\title{
SIRT3-mediated mitochondrial autophagy in refeeding syndrome-related myocardial injury in sepsis rats
}

\author{
Jiucui Li $^{1 \#}$, Kongmiao Lu ${ }^{1 \#}$, Xiao Zhang ${ }^{2 \#}$, Tianying Wang ${ }^{3}$, Qinghai Li $^{1}$, Xinjuan Yu ${ }^{3}$, Wei Han ${ }^{1}$, Lixin Sun ${ }^{2}$ \\ ${ }^{1}$ Department of Pulmonary and Critical Care Medicine, Qingdao Municipal Hospital, School of Medicine, Qingdao University, Qingdao, China; \\ ${ }^{2}$ Department of Anesthesiology, Qingdao Municipal Hospital, School of Medicine, Qingdao University, Qingdao, China; ${ }^{3}$ Clinical Research Center, \\ Qingdao Municipal Hospital, School of Medicine, Qingdao University, Qingdao, China \\ Contributions: (I) Conception and design: J Li, K Lu, X Zhang, L Sun, W Han; (II) Administrative support: W Han, Q Li; (III) Provision of study \\ materials or patients: T Wang, X Yu; (IV) Collection and assembly of data: X Yu; (V) Data analysis and interpretation: T Wang, L Sun; (VI) \\ Manuscript writing: All authors; (VII) Final approval of manuscript: All authors. \\ \#These authors contributed equally to this work. \\ Correspondence to: Wei Han. Department of Pulmonary and Critical Care Medicine, Qingdao Municipal Hospital, School of Medicine, Qingdao \\ University, Qingdao, Shandong, China. Email: hanw@qdu.edu.cn; Lixin Sun. Department of Anesthesiology, Qingdao Municipal Hospital, School of \\ Medicine, Qingdao University, Qingdao, Shandong, China. Email: Sunlixin1221@126.com.
}

\begin{abstract}
Background: Myocardial injury induced by refeeding syndrome (RFS) is one of the important causes of deterioration in critically ill patients. Sirtuin-3 (SIRT3) has been shown to regulate mitochondrial autophagy in myocardial ischemia/reperfusion injury; however, the role of mitochondrial autophagy on RFS-related myocardial injury in patients in critical condition has not been reported on.

Methods: Thirty Sprague-Dawley (SD) rats were divided into 3 groups ( $\mathrm{n}=10$ each group): the control group; the standard calorie refeeding (SCR) group; and the low calorie refeeding (LCR) group. The rats were weighed every third or four days from day 1 to day 14. On day 14, all rats were anesthetized and received an echocardiography test. Blood and bronchoalveolar lavage fluid (BALF) were collected and tested for arterial oxygen pressure $\left(\mathrm{PaO}_{2}\right)$, phosphorus $(\mathrm{P})$, and calcium $(\mathrm{Ca})$, creatine kinase-MB $(\mathrm{CK}-\mathrm{MB})$, lactate dehydrogenase (LDH), and cardiac troponin 1 (cTnI), myeloperoxidase (MPO), tumor necrosis factor $\alpha$ (TNF- $\alpha$ ), interleukin-1 $\beta$ (IL-1 $\beta$ ), and IL-6. The histopathological change of hearts and lungs were evaluated, and lung injury score was calculated. Mitochondrial autophagy related proteins (including Beclin1, LC3, mitofusin-2, Mfn2, PINK1, Parkin, and SIRT3) were analyzed using a Western blot. To evaluate the effect of SIRT3, 20 rats were divided into 2 groups ( $\mathrm{n}=10$ each group): The adeno-associated virus 9 (AAV9-Nc) group; and the AAV9-SIRT3 overexpression (AAV9-SIRT3) group. The protocols for rats were the same as the SCR group since day 22 after injection of AAV9. The protein expressions of PINK1, Parkin, and SIRT3 were compared between the AAV9-Nc group and AAV9-SIRT3 group.

Results: SCR caused significant decline in cardiac contractility and increased inflammatory cell infiltration in myocardial tissue. Meanwhile, Beclin1, LC3, PINK1, Parkin, and SIRT3 levels decreased, while Mfn2 showed no significant change. Furthermore, significant positive correlations were also found between SIRT3 and P, PINK1, and Parkin, and significant negative correlations were found between SIRT3 and CK-MB, LDH, and cTnI. Overexpression of SIRT3 activated the PINK1/Parkin mediated mitochondrial autophagy.

Conclusions: SIRT3 has an essential role in RFS-related myocardial injury during LPS induced chronic sepsis in rats, probably via regulating mitochondrial autophagy.
\end{abstract}

Keywords: Refeeding syndrome (RFS); myocardial injury; sirtuin-3 (SIRT3); mitochondrial autophagy; sepsis

Submitted Dec 22, 2021. Accepted for publication Feb 18, 2022.

doi: $10.21037 / \mathrm{atm}-22-222$

View this article at: https://dx.doi.org/10.21037/atm-22-222 


\section{Introduction}

Refeeding syndrome (RFS) is a potentially fatal clinical syndrome characterized by acute metabolic disorder after long-term hunger and re-eating. It is one of the important reasons for the deterioration of critical patients (1). Severe complications of RFS include acute arrhythmia, acute heart failure, Wernicke encephalopathy, rhabdomyolysis, and acute cardiac events $(2,3)$. In recent years, clinical practice has found that standards made by NICE (British National Institute for health and clinical technology optimization), which are widely used internationally to identify high-risk patients with RFS, are not suitable for early identification of RFS in critically ill patients (4). Our previous study (nct04005300) found that the incidence of RFS was about $30 \%$ in critically ill patients in China, and the incidence of acute cardiac events (acute heart failure and arrhythmia) in patients with RFS was twice as high as that in patients without RFS ( $27 \%$ vs. $12.6 \%)$ with increased mortality ( $26.7 \%$ vs. $11 \%)$. However, the mechanism of RFS is complicated by myocardial damage in critically ill patients and has not been clarified, and there is a lack of effective treatment.

The continuous contraction, relaxation, and electrical activity of cardiomyocytes requires continuous energy supply (5). Therefore, it is very important to maintain the homeostasis of mitochondria, which is the core of energy supply in cardiomyocytes (5). When critical ill patients were starving, autophagy was enhanced to maintain life activities, and the mitophagy was also enhanced due to excessive mitochondrial damage (6). Therefore, enhanced autophagy is an important protective biological behavior and is the survival mode of cardiomyocytes under conditions of starvation and stress (7). However, this seemingly reasonable feedback mechanism may bring risks to critically ill patients with RFS. The sudden decrease of the level of mitochondrial autophagy during RFS may lead to a large amount of damaged mitochondrial storage caused by stress and may result in energy metabolism disorder and the damage of cardiomyocytes.

As an important deacetylase family in vivo, the silent information regulation 2 family plays an important role in transcription regulation, energy metabolism regulation, cell survival, and DNA repair, and has become a research hotspot in recent years. In mammalian cells, the SIRT family has 7 members, which are named Sirtuin (SIRT) 1-7. SIRT1, 6, and 7 are mainly distributed in the nucleus to regulate the acetylation level of histone. SIRT2 is mainly distributed in the cytoplasm to regulate cell cycle and glucose metabolism.
SIRT3, 4, and 5 are mainly distributed in mitochondria to participate in autophagy and apoptosis (8).

Heart tissue is one of the organs most affected by SIRT3. After SIRT3 knockout, mitochondrial protein acetylation in cardiomyocytes increases. Excessive acetylation can lead to myocardial conduction dysfunction and abnormal myocardial rhythm, and can induce arrhythmia $(9,10)$. Zhang et al. found that the down regulation of SIRT3 in central myocytes of septic cardiomyopathy induced by lipopolysaccharide (LPS) led to excessive acetylation of key enzymes in the myocardial tricarboxylic acid cycle and also led to myocardial dysfunction (11). Previous studies have found that the increase of SIRT3 can activate mitochondrial autophagy and promote cell repair in a variety of disease models such as non-alcoholic fatty liver (12), myocardial infarction (13), and hypertensive heart disease (14). It is possible for the SIRT3 pathway to be involved in myocardial damage, however, the effects and mechanisms of SIRT3 and mitochondrial autophagy in RFS-related myocardial injury of sepsis rats is still unknown. Therefore, we used the LPS-induced sepsis model to explore the potential mechanisms of RFS-related myocardial injury in rats and to explore whether SIRT3 can become a potential prevention and treatment target of RFS myocardial injury in critically ill patients.

We present the following article in accordance with the ARRIVE reporting checklist (available at https://atm. amegroups.com/article/view/10.21037/atm-22-222/rc).

\section{Methods}

\section{Animals}

The healthy male specific pathogen free (SPF) SpragueDawley (SD) rats (5 rats/cage; 200-250 g each) aged 6-8 weeks were purchased from the Jinan Pengyue Experimental Animal Breeding Co., Ltd., Jinan, China. The rats were housed at $24{ }^{\circ} \mathrm{C}$ with 12 -h light-dark cycles and had free access to eat and drink. A protocol was prepared before the study without registration. All experimental procedures were approved by the ethics committee of Qingdao Municipal Hospital (approval No. 2021-116) and were in accordance with the Guide for the Care and Use of Laboratory Animals, 8th edition.

\section{Model establishment and grouping}

Animal selection, model preparation, sample preparation and analysis, and outcome processing were done by different 
experimenters. Animal selection, sample analysis, and outcome processing was performed with the experimenter blinded to the grouping.

First, 30 rats were assigned into 3 groups using the random number table method, with 10 rats in each group, as follows:

The control group (phosphate buffer saline, PBS ip + standard diet): All rats received an intraperitoneal injection of the corresponding amount of PBS at 7 o'clock on the first day. Based on previous studies $(15,16)$, the rats were randomized to receive enteral nutrition (EN) via duodenal papilla catheters $(1.2 \mathrm{~mm}$ epidural catheters). One end of catheter was inserted at $5 \mathrm{~cm}$ distal to the ligament of Treitz. The other end of the catheter was subcutaneously tunneled into the rat's back and properly secured. The detailed composition of the EN solution (Huarui Company, China) was as follows: whey protein isolate, maltose dextrin, vegetable oils, minerals, the trace element, and vitamins. EN was infused at a rate of $2 \mathrm{~mL} / \mathrm{h}$ for 10-12 minutes at the beginning. The total amount of final solution was allowed to reach and remain at $50 \mathrm{~mL} / 24 \mathrm{~h}$. Each rat received the energy of $30 \mathrm{Kcal} / 100 \mathrm{~g} / 24 \mathrm{~h}$, i.e., the standard diet for 14 days (17).

The standard calorie refeeding (SCR) group (LPS ip + standard diet): Rats received an intraperitoneal injection of LPS serotype O127:B7 (10 mg/kg, Sigma-Aldrich, UK) (18) at 7 o'clock on the first day. The duodenal papilla catheter placement was the same as above. EN was infused at a rate of $2 \mathrm{~mL} / \mathrm{h}$ for $10-12$ minutest at the beginning. The total amount of final solution was allowed to reach and remain at $50 \mathrm{~mL} / 24 \mathrm{~h}$. Each rat received the energy of $15 \mathrm{Kcal} / 100 \mathrm{~g} / 24$ h, i.e., half the standard diet for 7 days to establish a sepsis undernutrition critical condition. After 7 days, the body weight of each rat was less than $200 \mathrm{~g}$, indicating that the rat model of undernutrition was successful. Then, after the two days fasting, a standard diet $(30 \mathrm{Kcal} / 100 \mathrm{~g} / 24 \mathrm{~h}$ ) was applied for another 5 days.

The low calorie refeeding (LCR) group (LPS ip + escalated diet): The LPS treatment and catheter placement were done as described above, except that the refeeding rate and energy were increased in step-by-step at a rate of $10 \%$ of the fixed full diet until fixed full diet were applied (19).

The rats were weighed every 3 or 4 days from day 1 to day 14 . On day 14 , all rats received a routine echocardiography examination. Then, they were all euthanized with $\mathrm{CO}_{2}$ asphyxiation, and blood, bronchoalveolar lavage fluid (BALF), heart, and lung tissue samples were collected for correlation detection.

Second, 20 male SD rats were randomly divided into 2 groups ( $\mathrm{n}=10$ for each group), as follows:

The adeno-associated virus 9 group (AAV9-Nc group): Rats were injected with the corresponding AAV9 $(100 \mu \mathrm{L} / \mathrm{rat})$ through the tail vein. After 3 weeks of virus infection, rats were used to construct the SCR model (the method was the same as the detailed above SCR processing). The AAV9 was manufactured by Hanheng Biotechnology Co., Ltd., Shanghai, China. The virus titer was $5.0 \times 10^{11} \mu \mathrm{g} / \mathrm{mL}$.

The AAV9-SIRT3 overexpression group (AAV9-SIRT3 group): Rats were injected with the AAV9 vectors carrying overexpressing SIRT3 $(100 \mu \mathrm{L} / \mathrm{rat})$ through the tail vein. Experimental contents and methods were the same as the AAV9 group. The AAV9 vectors carrying overexpressing SIRT3 were manufactured by Hanheng Biotechnology Co., Ltd., Shanghai, China. The virus titer was $5.0 \times 10^{11} \mu \mathrm{g} / \mathrm{mL}$.

After all operations, rats were euthanized with $\mathrm{CO}_{2}$ asphyxiation. Correlation samples were taken for detection. No adverse events were observed during the experiment. The specific grouping is listed in the Figure 1.

\section{Echocardiography}

Before the rats were euthanized, all rats were anaesthetized with $1.5 \%$ isoflurane. Echocardiography (Sonos7500, $30 \mathrm{MHz}$ ) was performed periodically to assess the structure and cardiac systolic function of the heart (20).

\section{Blood gas analysis}

Blood samples ( $2 \mathrm{~mL}$ per rat) were obtained from the abdominal aorta using a blood collection needle. Plasma levels of arterial oxygen pressure $\left(\mathrm{PaO}_{2}\right)$, phosphorus $(\mathrm{P})$, and calcium $(\mathrm{Ca})$ levels were measured using a blood gas analyzer (Radiometer, Denmark).

\section{Enzyme linked immunosorbent assay (ELISA)}

As previously described (21), the trachea was intubated to collect BALF. One-sided lavage was performed with $2 \mathrm{~mL}$ of saline 3 times, and a total volume of $4.8 \mathrm{~mL}$ was recovered and centrifuged at 2,500 $\mathrm{g}$ for 10 minutes. BALF cytokine levels (tumor necrosis factor $\alpha$, TNF- $\alpha$, interleukin, IL-1 $\beta$, and IL-6) and myeloperoxidase (MPO) were analyzed with ELISA (Thermo Fisher Scientific, Waltham, MA, USA) on day 14. 


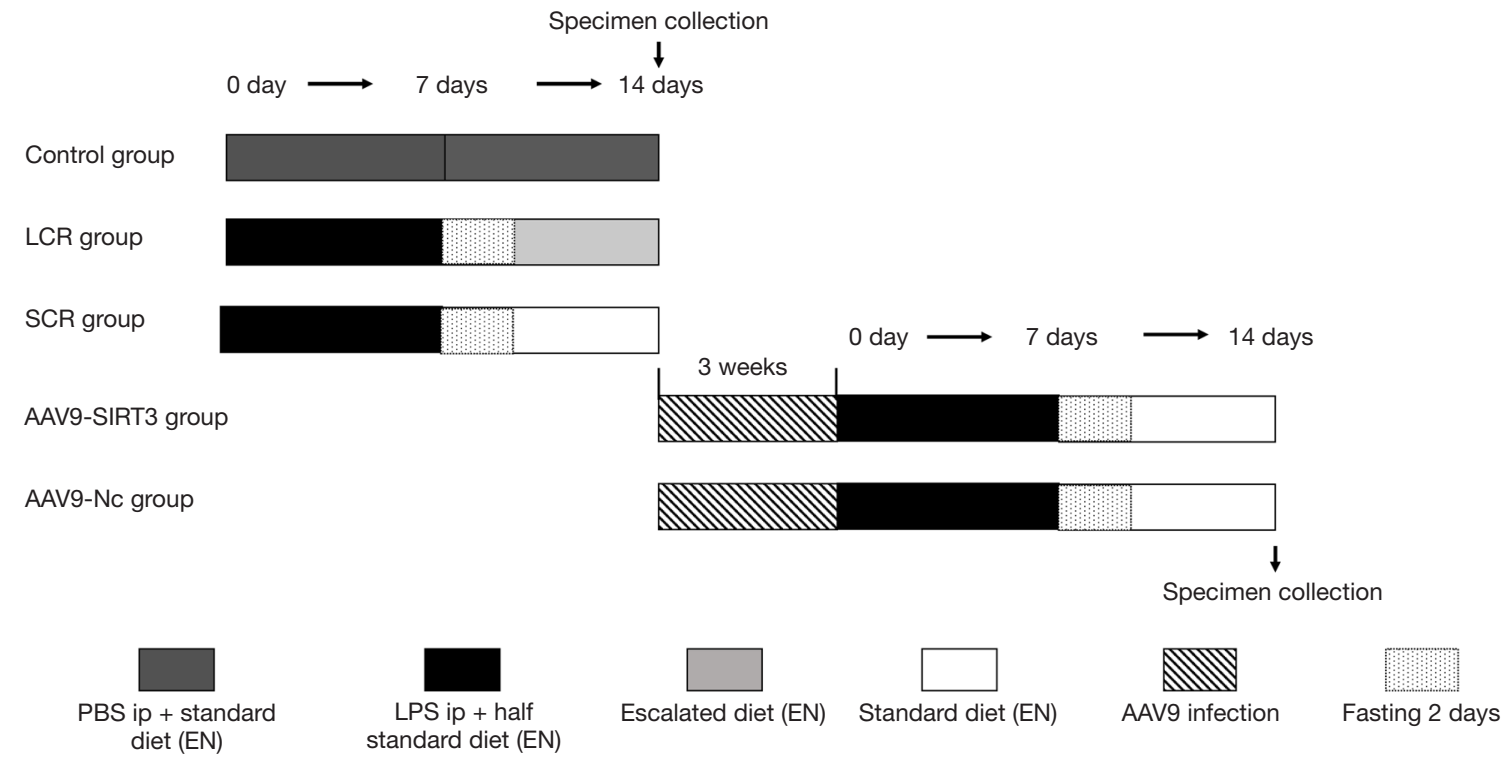

Figure 1 Model establishment. LCR, low calorie refeeding; SCR, standard calorie refeeding; AAV9-SIRT3 group, AAV9-SIRT3 overexpression group; AAV9-Nc group, adeno-associated virus 9 group; PBS, phosphate buffer saline; EN, enteral nutrition; LPS, lipopolysaccharide.

Blood ( $2 \mathrm{~mL})$, collected by heart puncture on day 14 , was centrifuged (1,500 rpm, 10 minutes) to obtain plasma to test the blood creatine kinase-MB (CK-MB), lactate dehydrogenase (LDH), and cardiac troponin 1 (cTnI). These were all determined using an ELISA kit (Thermo Fisher Scientific, Waltham, MA, USA).

\section{Histopathology}

The rat cardiac and lung tissue samples were sectioned and stained with hematoxylin-eosin staining (HE) staining using an HE kit (Beyotime Biotechnology, Shanghai, China) to evaluate histopathological changes. Cardiac and lung tissues were paraffin-embedded, dewaxed, and hydrated according to methods used in previous studies (22), and the images were analyzed using a light BX40 microscope (200× magnification) (Olympus Optical Company, Tokyo, Japan). Four histologic features (infiltration of inflammatory cells, alveolar hemorrhage, alveolar edema, and interstitial thickening) were included in the pathological evaluation of lung samples. Each feature was classified into 4 grades: absent, mild, moderate, or severe. A score of 0 to 3 was assigned based on the severity of the damage. The sums of scores were the lung injury score. The lung injury score was calculated by 2 different pathologists. We took the average scores if the 2 pathologists arrived at different.

\section{Western blot}

The remaining cardiac tissues were defrosted for a Western blot analysis. After the cardiac tissues was homogenized, an appropriate amount of radioimmunoprecipitation assay (RIPA) lysis buffer (Solarbio, Beijing, China) containing $0.1 \mathrm{mM}$ of phenylmethylsulfonyl fluoride (PMSF) was added. BCA kit (Elabscience Biotechnology Co. Ltd, Wuhan, China) was used to determine the protein concentration. The total protein sample was loaded into a $10 \%$ sodium dodecyl sulfatepolyacrylamide gel electrophoresis (SDS-PAGE) loading buffer before being transferred to polyvinylidene difluoride (PVDF) membranes (Millipore, USA). Protein samples were then incubated with specific antibodies against GAPDH (1:3,000, Aksomics Inc, KC-5G5), Cleaved Caspase-3 (1:250, Abcam, ab13847), Caspase-3 (1:500, CST, 8G10), SIRT3 (1:1,000, CST, 5490S), PINK1 (1:1,000, Abcam ab186303), Parkin (1:1,000, Abcam, ab77924), Beclin1 (1:1,000, Abcam, ab62472), LC3 (1:1,000, SIGMA, SAB1306269-40TST), and mitofusin-2 (Mfn2, 1:1,000, CST, \#9482) by drying overnight at $4{ }^{\circ} \mathrm{C}$ with gentle shaking. The GAPDH gene was used as the reference, and the expressions of specific proteins were normalized to GAPDH. The membranes were incubated for 1 hour with secondary antibodies (Abcam, USA): goat anti-mouse $\operatorname{IgG}(1: 5,000)$ and goat anti-rabbit $(1: 8,000)$ 
A

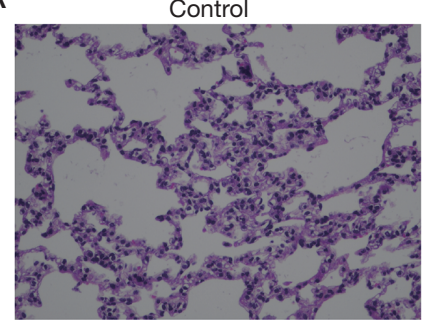

B

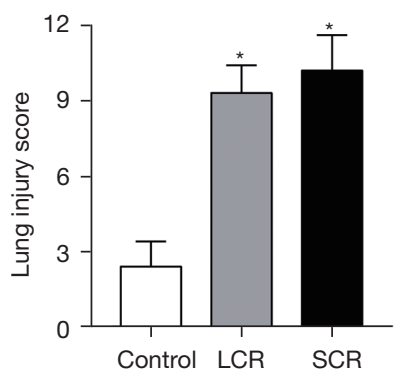

$E$

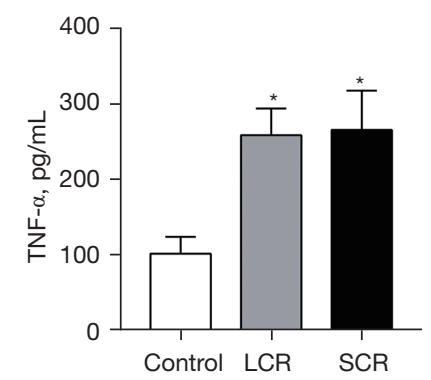

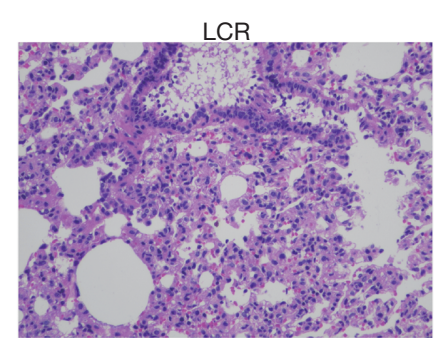

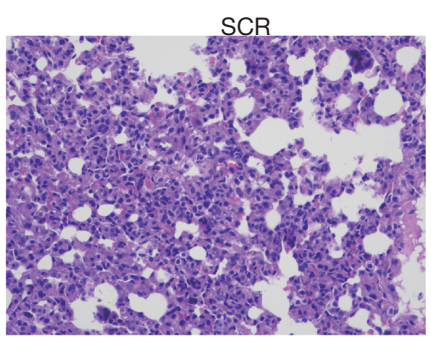

C

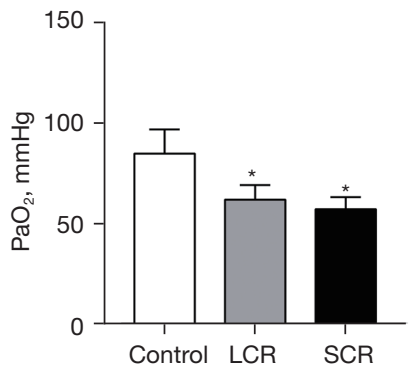

$\mathrm{F}$

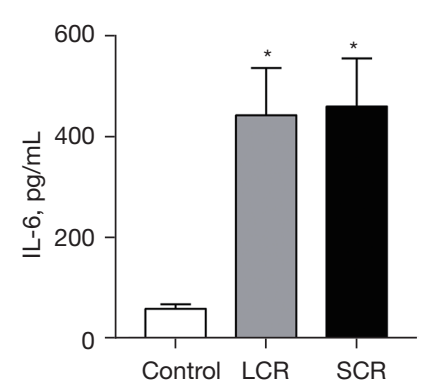

D

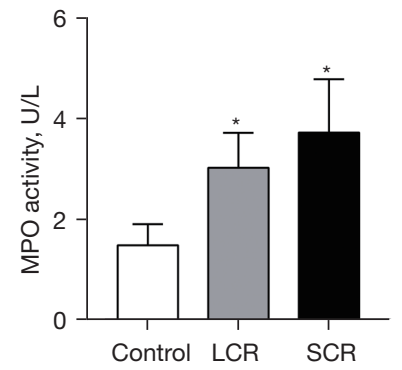

G

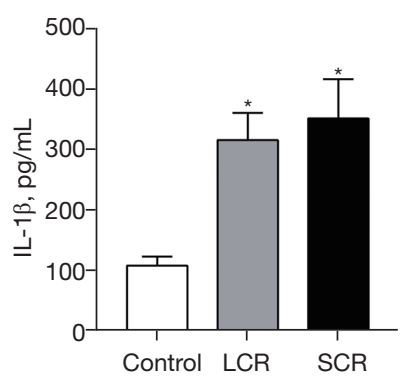

Figure 2 The rat chronic sepsis model was successfully established. (A) HE staining of lung tissue (200x magnification). (B) Lung injury score. (C) The arterial blood gas analysis of $\mathrm{PaO}_{2}$. (D) MPO. (E) TNF- $\alpha$. (F) IL-6. (G) IL-1 $\beta$. Data are presented as (n=10). *, P<0.05 compared with the control group. HE, hematoxylin-eosin; LCR, low calorie refeeding; SCR, standard calorie refeeding; $\mathrm{PaO}_{2}$, arterial oxygen pressure; MPO, myeloperoxidase; TNF- $\alpha$, tumor necrosis factor $\alpha$; IL-6, interleukin-6; IL-1 $\beta$, interleukin-1 $\beta$.

IRdye $^{\circledR} 800 \mathrm{CW}$ (LI-COR) IgG. Following enhanced chemiluminescence (ECL) reagent color development, ChampChemi 610 gel imaging system (Beijing Saizhi venture Technology Co., Ltd, Beijing, China) was used to scan the bands. Image J analysis software (NIH, Bethesda, MD, USA) was applied to read the gray value.

\section{Statistical analysis}

Statistical analysis was done using IBM SPSS Statistics 22 (IBM Corp, Armonk, NY, USA). Data are presented as mean \pm standard deviation (SD). The sample size was chosen based on previous studies of this kind (18). Significant differences between groups were estimated by an independent-samples $t$-test or One-Way ANOVA with
LSD post-hoc test. The associations between SIRT3 and other indicators were investigated by Pearson correlation analysis. $\mathrm{P}<0.05$ was considered statistically significant.

\section{Results}

\section{Rat chronic sepsis model and RFS model was successfully established}

Initially, the rat chronic sepsis model was successfully established by LPS. Alveolar edema, inflammatory cellular infiltration, and thickened septum were observed in the lung tissue of the SCR group by HE staining (Figure $2 A$ ). Compared with the control group, the lung injury score and the arterial blood gas analysis of $\mathrm{PaO}_{2}$ were lower in 

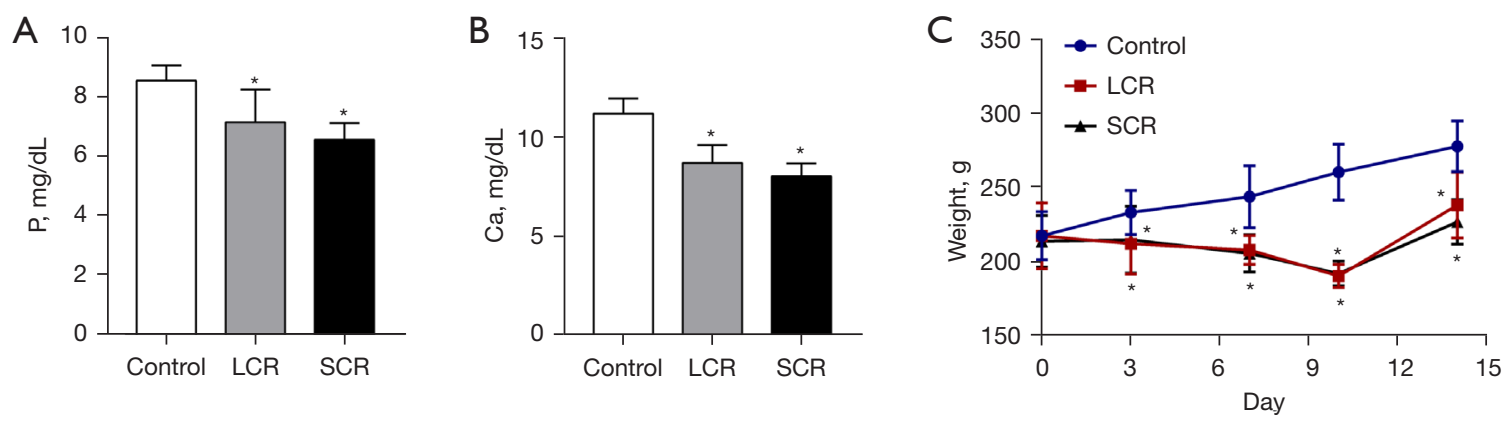

Figure 3 The RFS model was successfully established. (A) Blood P levels. (B) Blood Ca levels. (C) The body weight change (every third or four days from day 1 to day 14$)$ of rats. Data are presented as mean $\pm \mathrm{SD}(\mathrm{n}=10){ }^{*}, \mathrm{P}<0.05$ compared with the control group. RFS, refeeding syndrome; LCR, low calorie refeeding; SCR, standard calorie refeeding; P, phosphorus; Ca, calcium.

the SCR group (Figure 2B,2C). However, the indicators mentioned above did not reach statistical significance between the SCR group and the LCR group. Moreover, as shown in Figure 2, LPS significantly increased TNF- $\alpha$, IL-1 $\beta$, IL-6, and MPO levels throughout this study. These positive results indicated that the rat chronic sepsis model was successfully established. To investigate whether the RFS model had been successfully induced, blood electrolytes ( $\mathrm{P}$ and $\mathrm{Ca}$ ) and body weight (every 3 or 4 days from day 1 to day 14) of rats were measured. As expected, blood $\mathrm{P}$ and Ca levels in the SCR group and the LCR group decreased compared to the control group. Although these differences of P and Ca between the SCR group and the LCR group did not reach statistical significance, there was an apparent trend towards a difference (Figure $3 A, 3 B$ ). After half daily diet for 7 days, the body weight of rats in the SCR and the LCR group were decreased. Following 7 days of refeeding, the body weight of rats in the SCR and the LCR group caught up with that of the control group (Figure 3C). Although the body weight change was not statistically significant between the SCR group and the LCR group, this difference persisted throughout the study. These results demonstrated that the rat RFS model was successfully established.

\section{Myocardial injury occurred in RFS}

The model of RFS was carried out to explore the mechanism of impaired myocardium. We classified the model into two groups (LCR and SCR) following the calorie percentage for the moments of refeeding. All rats in the 2 groups were examined using a cardiac ultrasound (Figure 4A). Myocardial tissue was collected for myocardial pathological examination and molecular biology examination. Our initial findings showed a decline in cardiac contractility of the SCR group as envisioned, compared to LCR group. In the SCR group, the pathological examination of samples showed the structure of cardiac tissue was disordered with increased infiltration of inflammatory cells (Figure $4 A, 4 B$ ). The significantly increased levels of Cleaved Caspase- 3 in the LCR group detected by Western blot indicated that the cardiac tissue was undergoing apoptosis (Figure $4 C, 4 D$ ). In addition, we observed that blood LDH, CK-MB, and cTnI levels increased after SCR (Figure 4E-G). Along with LCR, blood $\mathrm{LDH}, \mathrm{CK}-\mathrm{MB}$, and cTnI levels showed reductions, showing that LCR may alleviate RFS-related myocardial damage.

\section{Autophagy inhibition in myocardial tissue induced by standard calorie refeeding}

The expression of autophagy-related proteins (Beclin1 and LC3) detected by Western blot was used to assess the role of autophagy myocardial tissue induced by SCR. As shown in Figure 5, in the SCR group, Beclin1 and LC3 had decreased expression compared to that in the LCR group, which suggested that autophagy was reduced in the SCR group.

\section{Mfn2, PINK1, Parkin, and SIRT3 expression were decreased in myocardial tissue following standard calorie refeeding}

No significant change was detected in Mfn2 (Figure 6A,6B). Standard calorie refeeding decreased the expression of PINK1, Parkin, and SIRT3 in myocardial tissue from SD rats compared with the LCR group (Figure 6C-E). This observation implied that mitochondrial autophagy was 


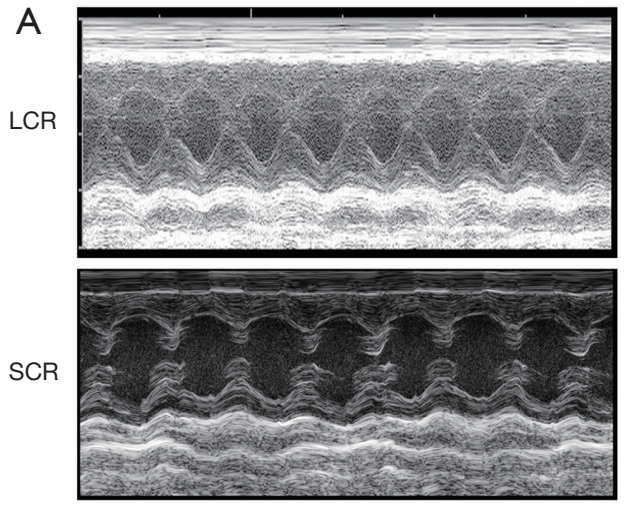

$\mathrm{E}$

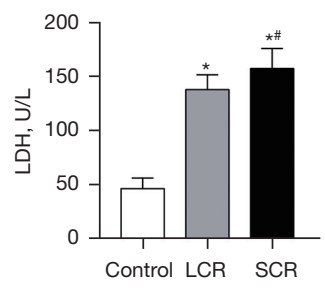

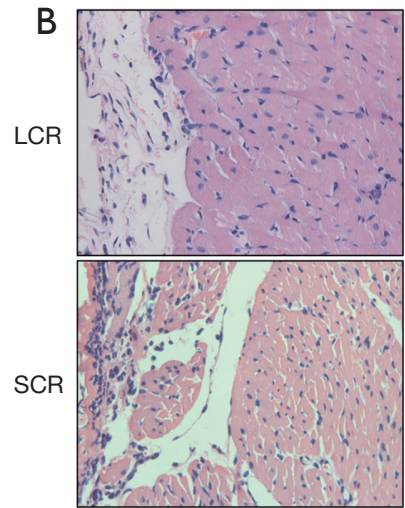

$\mathrm{F}$

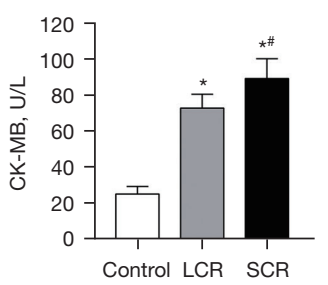

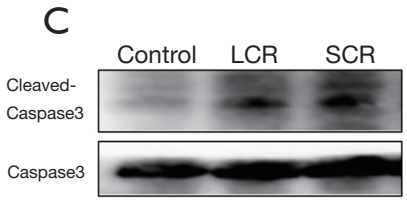

$\mathrm{D}$

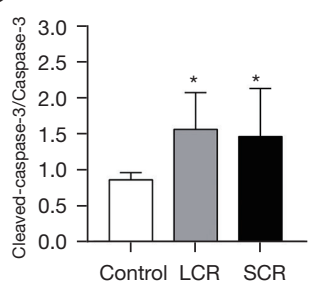

G

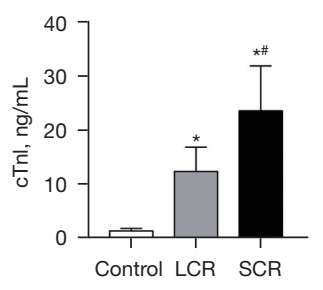

Figure 4 Myocardial injury occurred in the RFS model. (A) Two-dimensional echocardiography images. (B) HE staining of myocardial tissue (100× magnification). (C,D) The relative expression levels of Caspase-3 and Cleaved Caspase-3. (E) LDH. (F) CK-MB. (G) cTnI. Data are presented as mean $\pm \mathrm{SD}(\mathrm{n}=10)$. *, $\mathrm{P}<0.05$ compared with the control group; ${ }^{*}, \mathrm{P}<0.05$ compared with LCR group. RFS, refeeding syndrome; LCR, low calorie refeeding; SCR, standard calorie refeeding; HE, hematoxylin-eosin; LDH, lactate dehydrogenase; CK-MB, creatine kinase-MB; cTnI, cardiac troponin 1.

A

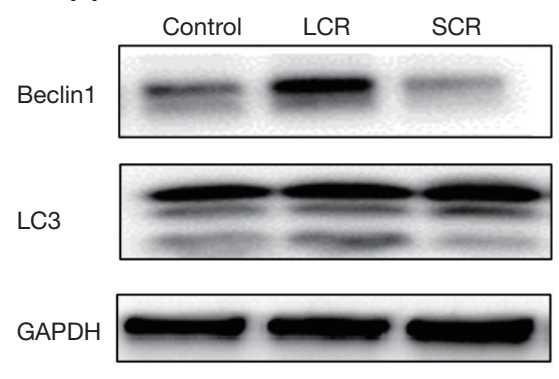

B

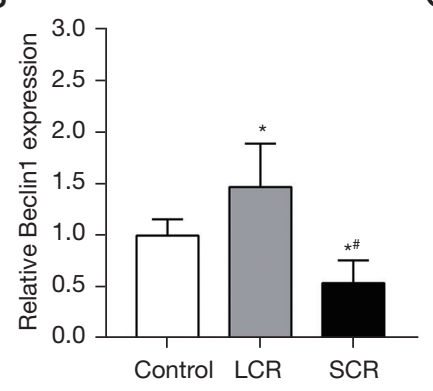

C

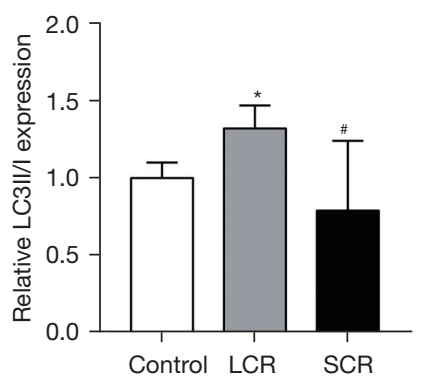

Figure 5 Autophagy inhibition in myocardial tissue induced by SCR. (A) Western blot assay for Beclin1 and LC3 protein expression. (B) Statistic analysis of Beclin1 protein expression. (C) Statistic analysis of LC3II/I protein expression. Data are presented as mean $\pm \mathrm{SD}(\mathrm{n}=10)$. *, $\mathrm{P}<0.05$ compared with the control group; *, $\mathrm{P}<0.05$ compared with LCR group. LCR, low calorie refeeding; SCR, standard calorie refeeding.

reduced.

\section{Correlation analysis of SIRT3 with LDH, CK-MB, cTnI, $P$, Ca, PINK1, and Parkin}

The associations between SIRT3 and LDH, CK-MB,
cTnI, P, Ca, PINK1, and Parkin were investigated by Pearson correlation analysis. The relative expression of SIRT3 showed medium negative correlations with LDH $(\mathrm{r}=-0.537 ; \mathrm{P}=0.015), \mathrm{CK}-\mathrm{MB}(\mathrm{r}=-0.527 ; \mathrm{P}=0.017)$, and cTnI concentration $(\mathrm{r}=-0.644 ; \mathrm{P}=0.002)$ (Figure $7 A-C$ ), medium positive correlations with $\mathrm{P}(\mathrm{r}=0.509 ; \mathrm{P}=0.022)$, 

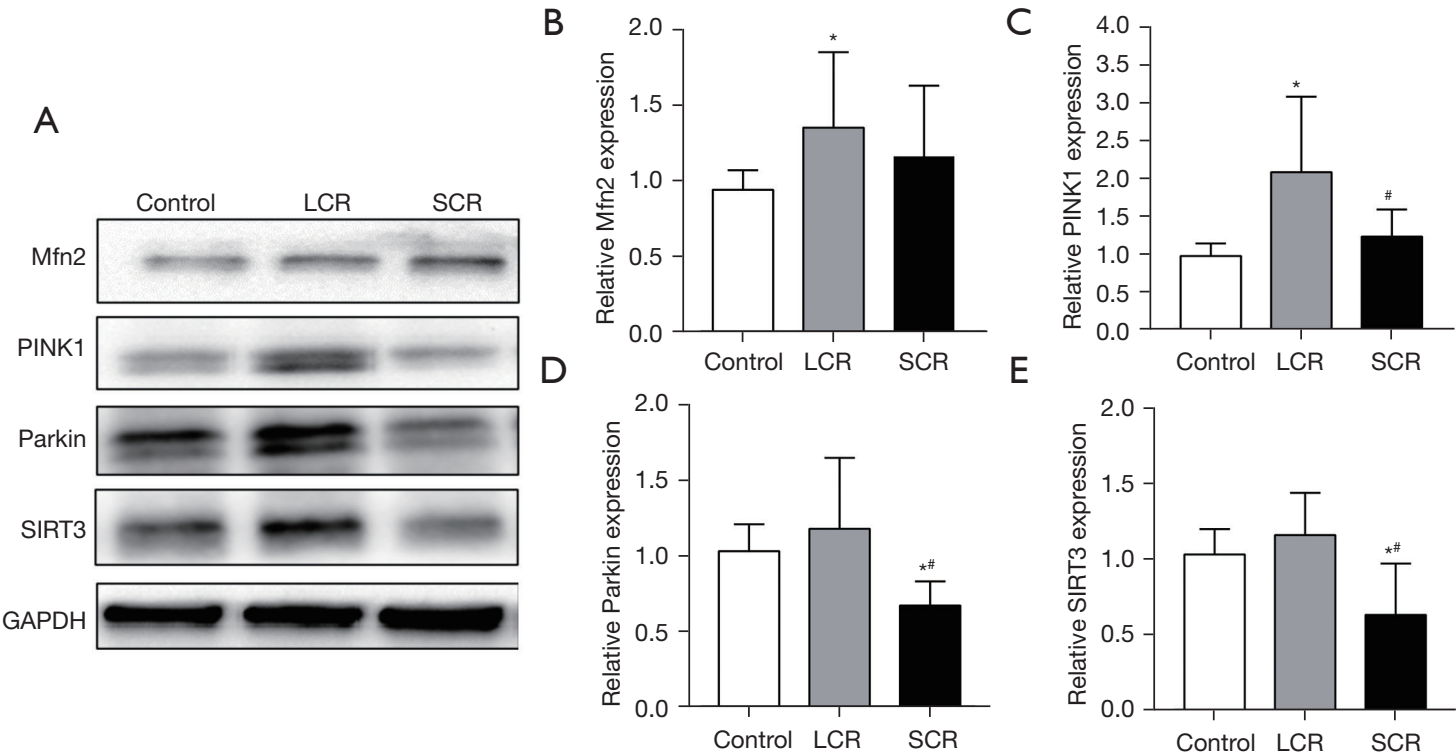

Figure 6 PINK1, Parkin, and SIRT3 expression were decreased in myocardial tissue following standard calorie refeeding (A) Western Blot assay for Mfn2, PINK1, Parkin, and SIRT3 protein expression. (B) Statistic analysis of Mfn2 protein expression (C) Statistic analysis of PINK1 protein expression. (D) Statistic analysis of Parkin protein expression. (E) Statistic analysis of SIRT3 protein expression. Data are presented as mean $\pm \mathrm{SD}(\mathrm{n}=10) .{ }^{*}, \mathrm{P}<0.05$ compared with the control group; ${ }^{\#}, \mathrm{P}<0.05$ compared with the LCR group. LCR, low calorie refeeding; SCR, standard calorie refeeding; SIRT3, Sirtuin 3; Mfn2, mitofusin-2.

PINK1 $(\mathrm{r}=0.598 ; \mathrm{P}=0.005)$, and Parkin $(\mathrm{r}=0.676 ; \mathrm{P}=0.001)$ (Figure $7 D-F)$ and no correlation $(\mathrm{r}=0.393, \mathrm{P}=0.086)$ with $\mathrm{Ca}$ in the LCR + SCR group.

\section{Effect of SIRT3 overexpression on mitochondrial autophagy (PINK1/Parkin signaling pathway)}

A significant correlation was observed between SIRT3 and PINK1/Parkin. To verify the relationships, in vivo, AAV9SIRT3 was delivered to adult rats before establishing the RFS model in order to determine the function of SIRT3 overexpression. The results showed that SIRT3 overexpression led to an increase in PINK1 and Parkin expression in myocardial tissue (Figure 8). This indicated that the PINK1/Parkin signaling pathway was mediated by SIRT3.

\section{Discussion}

RFS is common in patients with severe infection requiring intensive care. In this study, the pathogenesis of RFS-related myocardial injury in patients with sepsis were investigated. SIRT3 and PINK1/Parkin signaling pathways were shown to have key molecular roles in RFS-related myocardial injury. To the best of our knowledge, this study described for the first time an RFS model in sepsis rats.

RFS is a potentially life-threatening clinical syndrome that may occur in malnourished patients (1). Hypophosphatemia and RFS are frequent and need to be avoided because of they cause morbidity. Reported morbidity and mortality rates in critically ill patients with RFS have reached approximately $34-52 \%$ and $22 \%$, respectively in 13 hospital intensive care units (ICUs) in Australia and New Zealand $(23,24)$. However, no reliable animal model has been able to mimic the characteristics of RFS-related myocardial injury in sepsis patients. The existing RFS animal models are not made to represent critical illness and cannot simulate RFS under critically ill conditions. Therefore, it is urgent to establish an efficient RFS animal model that meets clinical needs for the study of myocardial injury. In this study, the refeeding strategy was performed on the basis of sepsis. As one of the most common critical illnesses in the ICU, sepsis and sepsisassociated nutritional management remain a difficult problem to solve (25). The pathology of lung injury and elevated plasma TNF- $\alpha$, IL- $1 \beta$, IL- 6 , and MPO demonstrated that our sepsis model was well-constructed.

Nutritional support in patients with sepsis has become an 

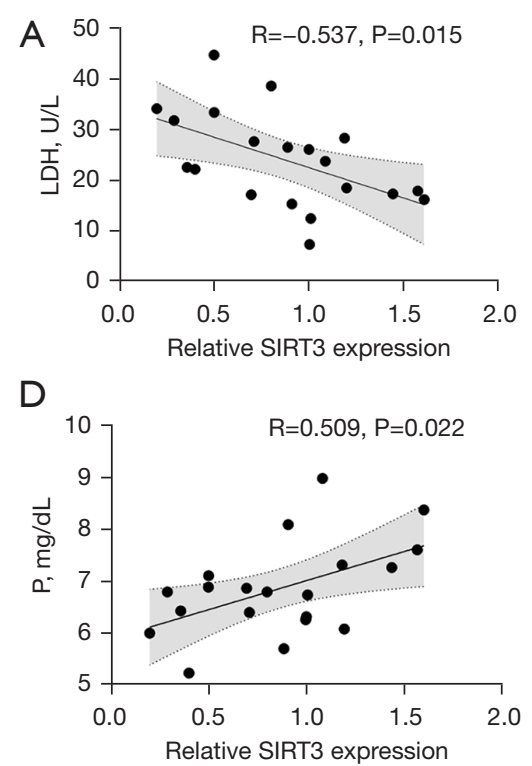

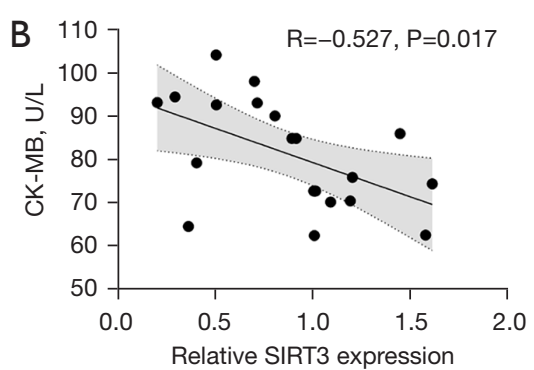

E

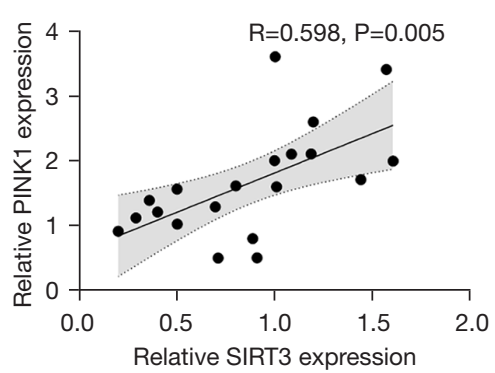

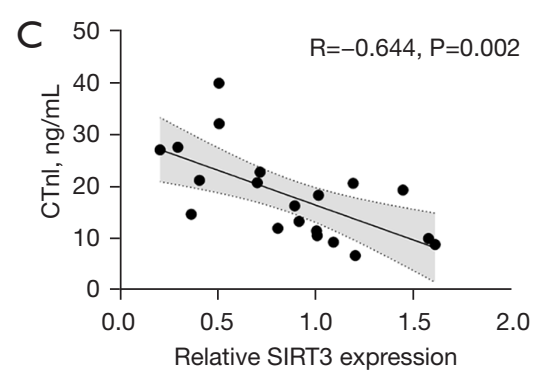

$\mathrm{F}$

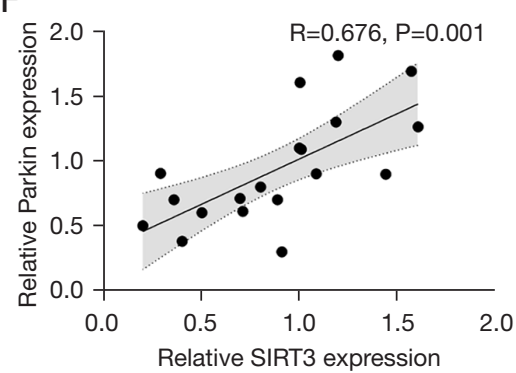

Figure 7 Correlation analysis of SIRT3 with LDH, CK-MB, cTnI, P, PINK1, and Parkin in the LCR + SCR group. A moderate negative correlation was observed between SIRT3 and LDH (A), CK-MB (B), and cTnI (C). A medium positive correlation was observed between SIRT3 and P (D), PINK1 (E), and Parkin (F). LCR, low calorie refeeding; SCR, standard calorie refeeding; SIRT3, Sirtuin 3; LDH, lactate dehydrogenase; CK-MB, creatine kinase-MB; cTnI, cardiac troponin 1; P, phosphorus.

A

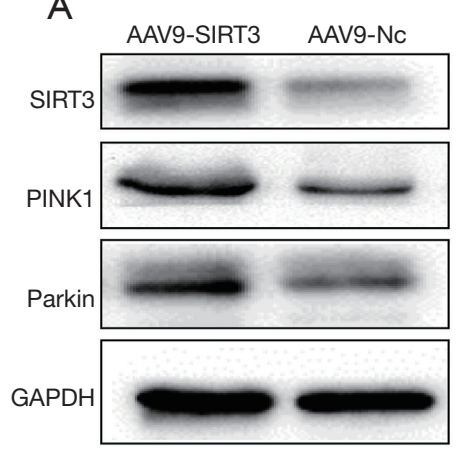

B

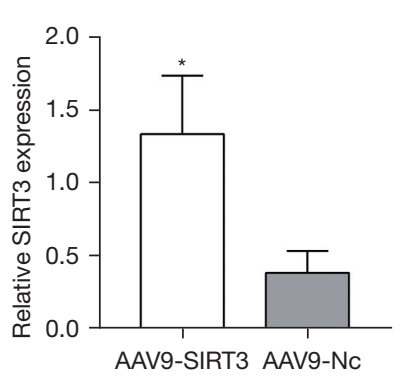

C

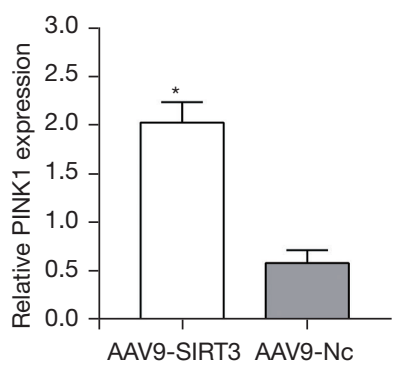

D

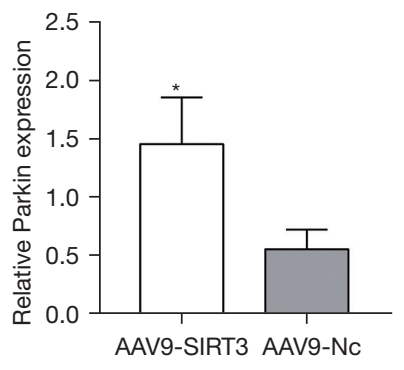

Figure 8 The effect of SIRT3 overexpression on mitochondrial autophagy (PINK1/Parkin signaling pathway) (A) Western blot assay for SIRT3, PINK1, and Parkin protein expression. (B) Statistic analysis of SIRT3 protein expression. (C) Statistic analysis of PINK1 protein expression. (D) Statistic analysis of Parkin protein expression. Data are presented as mean $\pm \mathrm{SD}(\mathrm{n}=10)$. * $\mathrm{P}<0.05$ compared with the AAV9Nc group. AAV9-SIRT3 group, AAV9-SIRT3 overexpression group; AAV9-Nc group, adeno-associated virus 9 group; SIRT3, Sirtuin 3.

integral part of modern surgery, which is considered to be one of the most significant advances of the 20th century (26). Early EN can prevent the cell metabolism disorder caused by the aggravation of trauma that is a result of the lack of nutrient substrate, and it can increase serum gastrin level and promote the movement of gastrointestinal mucosa, therefore early EN has extensive uses for critically ill patients. RFS is a common phenomenon in sepsis patients being re-fed EN. It is a potentially fatal clinical syndrome characterized by acute metabolic disorders that occurs after hunger and high calorie refeeding (27). Currently, almost all existing RFS models are either time consuming (the food intake of each rat needs to be calculated by subtracting the remaining food and excrement from its initial weight) (28), high cost (rats were refed with two fresh pollen-enriched diets) (29), or tedious (electrolytes/ minerals intake per day for rats were calculated exactly) (30). 
We developed a new RFS animal model using LPS ip and diet control. This kind of calorie regulation model seems to be more reflective of RFS in hospitalized patients. A fixed full diet ( $30 \mathrm{Kcal} / 100 \mathrm{~g} / 24 \mathrm{~h}$ ) mediated rat RFS model was established on the basis of the chronic sepsis in our study. A restricted caloric intake ( $<50 \%$ of resting energy expenditure) was used to prevent organ damage induced by refeeding (31). The calorie intake was increased $10 \%$ to $25 \%$ per day for one week until it reached the calorie goal (19). Therefore, escalated half diet is needed to determine whether such clinical intervention will be efficacious. Electrolyte disturbance, represented by and hypophosphatemia is a hallmark of RFS $(32,33)$. Glucose entry is accompanied by potassium, phosphorus, calcium, among others entry following refeeding after food deprivation, hence this reduced the amount of electrolytes. In our study, although not statistically significant, hypophosphatemia levels appeared to increase after the escalated half diet was applied, which suggested that this escalated method was effective at alleviating RFS.

In patients with RFS, comorbidities are common, with arrhythmias and rapid heart failure being the most common (2). There are very few reports that explore the pathogenesis of RFS-related myocardial injury. Ventricular arrhythmias are often observed in sepsis patients with RFS (3), which indicates potential myocardial injury. Moreover, the mechanism of RFS-related myocardial injury remains to be elucidated. It has been reported that CK$\mathrm{MB}, \mathrm{LDH}$, and cTnI are markers of myocyte injury (34), and myocardial apoptosis is also a prognostic indicator of myocardial injury (35). Appearance of a reduction in cardiac contractility following a diffuse multifocal inflammatory infiltrate, myocardial fiber disorganization, cardiomyocyte apoptosis, and increased expression of CK-MB, LDH, and $\mathrm{c} T \mathrm{TI}$ in our study indicated the occurrence of severe myocardial injury induced by SCR.

The myocardial tissue has a great demand for energy. Some experiments confirmed that changes in ATP levels caused autophagy level changes to ensure the stability of cell energy metabolism $(36,37)$. Autophagy exerts different effects in different pathological conditions of the heart for the current study. For example, autophagy can exert a harmful effect at myocardial ischemia-reperfusion (I/R) and pressure overload (38), whereas it can exert a beneficial effect in chronic myocardial infarction and diabetic cardiomyopathy (39). It was worth attending that the longterm severe "starvation" and refeeding inhibited autophagy instead clinically (6), possibly because ketone bodies could act as a substitute for autophagy to provide energy for cells. In our research, with the onset of standard calorie refeeding, the capability for intracellular debris to be removed and for new energy to be generated, that is autophagy, were significantly decreased, which led to irreversible myocardial damage in autophagy-related protein expression and indirectly reflected autophagy activity. Beclin1 expression level and the ratio of LC3-II/I were significantly positively related to autophagy, which somewhat can reflect the autophagy reactivity $(40,41)$.

At the molecular level, mitochondrial dysfunction, in combination with insufficient energy supply, reduces cardiomyocyte contractility, leading to myocardial injury caused by sepsis (42). Specifically, mitochondrial autophagy was thought to be beneficial for myocardial injury considering its role of degrading damaged mitochondria and clearing hazardous substances (43). Mitochondrial division appears to promote mitochondrial clearance to a certain extent, whereas fusion inhibits mitochondrial autophagy. In mitochondrial dynamics, a group of kineticrelated sGTPases are involved in mitochondrial division and fusion. During the fusion process, outer membrane fusion is followed by Mfn2 located in the mitochondrial outer membrane. Mfn2 expression aggravates abnormal mitochondrial function/dynamics, which disrupts fission and fusion balance (44). High networked mitochondria and a high expression level of Mfn2 have been identified in myocardial tissues with high-energy demands. This suggested that the Mfn2 protein was involved in the regulation of mitochondrial energy metabolism. Here, we reported no obvious alterations in Mfn2 expression in the SCR rats. These data suggested that no significant decrease was found for the fusion ability of mitochondria, therefore, the mitochondrial autophagy was inhibited. The PINK1/Parkin signaling pathway that mediated mitochondrial autophagy was most prevalent. PINK1 belongs to the serine/threonine kinase family and Parkin is an E3 ubiquitin ligase. Parkin is generally located in the cytoplasm under physiological conditions, and the protein activity is naturally inhibited (45). Long-term exposure to inflammation, oxidative stress, and other reactions can lead to drops of mitochondrial membrane potential and depolarization of the mitochondria. Following this, PINK1 could transfer to the depolarized mitochondrial outer membrane, cause Parkin phosphorylation by PINK1 to mediate ubiquitination, and participate in the process of mitochondrial autophagy. Accumulation and activation of PINK1 on the outer membrane of mitochondria is regarded 
as necessary to clear dysfunctional mitochondria (46). In addition, PINK1 and Parkin are associated with myocardial injury, and are known to be involved in myocardial I/R injury development (47). Our results showed decreases in the expression of PINK1 and Parkin in the SCR group, which indicated that mitochondrial autophagy decreased. This is consistent with the change of Mfn2 in our study. Collectively, PINK1/Parkin mediated mitochondrial autophagy played a protective role against RFS-related myocardial injury. When mitochondrial autophagy was inhibited, impaired mitochondrial accumulated and energy levels decreased.

The SIRT family is an important deacetylase family, which plays an important role in transcription regulation, energy metabolism regulation, cell survival, and DNA repair (48). SIRT3 is mainly distributed in heart (49). Studies have indicated that SIRT3-overexpressing O-hMSCs after myocardial injury enhanced cardiac resistance and function, which elucidated the protective role of SIRT3 in cardiomyocytes during myocardial infarction (11). In addition, emerging research has reported that SIRT3, a member of class III HDACs enriched in mitochondria, is involved in maintaining the organismal homeostasis, such as ATP production, ROS scavenging, and autophagy regulation (50). Increased SIRT3 expression prevents Superoxide Dismutase 2 (SOD2) hyperacetylation, reduces mitochondrial oxidative stress and vascular inflammation, thereby protecting vascular function and reducing hypertension. Besides, SIRT3 can regulate mitochondrial function by reducing mitochondrial permeability transition pore (mPTP) opening and transforming growth factor (TGF)- $\beta 1$ signaling $(10,51)$. Studies have shown that SIRT3 has a role in regulating mitochondrial autophagy in multiple diseases, including diabetic cardiomyopathy, myocardial injury, and heart failure $(52,53)$. In our study, SIRT3 expression level in the cardiac tissue of the SCR group was decreased compared with the LCR group. This pointed to the important role of SIRT3 in RFS-related myocardial injury. Regarding the current study, we also found mitochondrial autophagyrelated proteins (such as PINK1, Parkin, Mfn2) were simultaneously down-regulated. Recent investigations indicated that the upregulation of mitochondrial autophagy induced by PINK1/Parkin pathway was considered to be related to myocardial I/R injury (50). However, whether SIRT3 participates in the regulation of PINK1/ Parkin mediated mitochondrial autophagy in RFS- related myocardial injury remains unknown. In our study, correlation analyses indicated that SIRT3 and PINK1/ Parkin displayed a significant positive relationship, while they had a significant negative relationship with CK-MB, $\mathrm{LDH}$, and cTnI. These analyses indicated a relationship among SIRT3, PINK1/Parkin, and myocardial injury, but not the causal nature of this link. To support this link, we overexpressed SIRT3 in the SCR model by the AAV9 vectors transfection and observed the expression of PINK1 and Parkin. Our study found that over-expression of SITR3 led to increased PINK1/Parkin expression. These results suggest that down-regulated SIRT3 in the rat RFS model may modulate myocardial injury by suppressing the PINK1/Parkin pathway. Adenine nucleotide translocator 1 (ANT1) is a key molecule for the exchange of ADP and ATP through the inner mitochondrial membrane, which protects PINK1 from degradation and maintains normal mitochondrial autophagy by binding TIM44. While ANT1 was knocked out, TIM44 was less bound to ANT1 leading to PINK1 degradation. Meanwhile, decreased SIRT3 can lead to enhanced acetylation of CypD and promote the binding of CypD to ANT1, thus resulting in downregulation of ANT1 function and inducing mitochondrial damage $(54,55)$. Therefore, we speculate that the mechanism by which SIRT3 regulates the PINK1/Parkin pathway may be related to ANT1. This warrants further work to determine the detailed mechanisms.

Due to the limited observation period, we only observed changes of myocardial function, mitochondrial biogenesis and autophagy at a certain point in time, not dynamic changes at multiple time-points and cannot clarify the dynamical changes in RFS of sepsis rats. More research into the effects of autophagy and SIRT3 is required in the future. This is also the limitation of this experiment.

\section{Conclusions}

Taken together, our study described for the first time a model of RFS model in sepsis rats. By using this model, we found that SIRT3 participated in myocardial injury by regulating PINK1/Parkin mediated mitochondrial autophagy.

\section{Acknowledgments}

Funding: This work was supported by National Natural Science Foundation of China (Grant Nos. 81973012, 
81900048 and 82000051).

\section{Footnote}

Reporting Checklist: The authors have completed the ARRIVE reporting checklist. Available at https://atm. amegroups.com/article/view/10.21037/atm-22-222/rc

Data Sharing Statement: Available at https://atm.amegroups. com/article/view/10.21037/atm-22-222/dss

Conflicts of Interest: All authors have completed the ICMJE uniform disclosure form (available at https://atm. amegroups.com/article/view/10.21037/atm-22-222/coif). The authors have no conflicts of interest to declare.

Ethical Statement: The authors are accountable for all aspects of the work in ensuring that questions related to the accuracy or integrity of any part of the work are appropriately investigated and resolved. All experimental procedures were approved by the ethics committee of Qingdao Municipal Hospital (approval No. 2021-116) and were in accordance with the Guide for the Care and Use of Laboratory Animals, 8th edition.

Open Access Statement: This is an Open Access article distributed in accordance with the Creative Commons Attribution-NonCommercial-NoDerivs 4.0 International License (CC BY-NC-ND 4.0), which permits the noncommercial replication and distribution of the article with the strict proviso that no changes or edits are made and the original work is properly cited (including links to both the formal publication through the relevant DOI and the license). See: https://creativecommons.org/licenses/by-nc-nd/4.0/.

\section{References}

1. Wirth R, Diekmann R, Janssen G, et al. Refeeding syndrome : Pathophysiology, risk factors, prevention, and treatment. Internist (Berl) 2018;59:326-33.

2. Giovinazzo S, Sukkar SG, Rosa GM, et al. Anorexia nervosa and heart disease: a systematic review. Eat Weight Disord 2019;24:199-207.

3. Crook MA. Cardiac abnormalities in the refeeding syndrome. Nutrition 2017;35:146-7.

4. Arabi YM, Al-Dorzi HM. Trophic or full nutritional support? Curr Opin Crit Care 2018;24:262-8.

5. Zhou H, Ren J, Toan S, et al. Role of mitochondrial quality surveillance in myocardial infarction: From bench to bedside. Ageing Res Rev 2021;66:101250.

6. van Zanten ARH. Changing paradigms in metabolic support and nutrition therapy during critical illness. Curr Opin Crit Care 2018;24:223-7.

7. Lu J, Boheler KR, Jiang L, et al. Polycystin-2 Plays an Essential Role in Glucose Starvation-Induced Autophagy in Human Embryonic Stem Cell-Derived Cardiomyocytes. Stem Cells 2018;36:501-13.

8. Ding RB, Bao J, Deng CX. Emerging roles of SIRT1 in fatty liver diseases. Int J Biol Sci 2017;13:852-67.

9. Xu Y, Zhang S, Rong J, et al. Sirt3 is a novel target to treat sepsis induced myocardial dysfunction by acetylated modulation of critical enzymes within cardiac tricarboxylic acid cycle. Pharmacol Res 2020;159:104887.

10. Dikalova AE, Pandey A, Xiao L, et al. Mitochondrial Deacetylase Sirt3 Reduces Vascular Dysfunction and Hypertension While Sirt3 Depletion in Essential Hypertension Is Linked to Vascular Inflammation and Oxidative Stress. Circ Res 2020;126:439-52.

11. Zhang DY, Zhang CF, Fu BC, et al. Sirtuin3 protects aged human mesenchymal stem cells against oxidative stress and enhances efficacy of cell therapy for ischaemic heart diseases. J Cell Mol Med 2018;22:5504-17.

12. Li R, Xin T, Li D, et al. Therapeutic effect of Sirtuin 3 on ameliorating nonalcoholic fatty liver disease: The role of the ERK-CREB pathway and Bnip3-mediated mitophagy. Redox Biol 2018;18:229-43.

13. Zhao D, Sun Y, Tan Y, et al. Short-Duration Swimming Exercise after Myocardial Infarction Attenuates Cardiac Dysfunction and Regulates Mitochondrial Quality Control in Aged Mice. Oxid Med Cell Longev 2018;2018:4079041.

14. Wei T, Huang G, Gao J, et al. Sirtuin 3 Deficiency Accelerates Hypertensive Cardiac Remodeling by Impairing Angiogenesis. J Am Heart Assoc 2017;6:006114.

15. Wan S, Yang J, Mamtawla G, et al. Differential Metabolomic Analysis of Liver Tissues from Rat Models of Parenteral Nutrition-Associated Liver Disease. Biomed Res Int 2020;2020:9156359.

16. Kuçi O, Verlaan D, Vicente C, et al. Citrulline and muscle protein homeostasis in three different models of hypercatabolism. Clin Nutr 2020;39:917-27.

17. Peng L, Wu LG, Li B, et al. Early enteral nutrition improves intestinal immune barrier in a rat model of severe acute pancreatitis. J Hepatobiliary Pancreat Sci 2016;23:681-7.

18. Kirk RA, Kesner RP, Wang LM, et al. Lipopolysaccharide exposure in a rat sepsis model results in hippocampal 
amyloid- $\beta$ plaque and phosphorylated tau deposition and corresponding behavioral deficits. Geroscience 2019;41:467-81.

19. Stanga Z, Brunner A, Leuenberger M, et al. Nutrition in clinical practice-the refeeding syndrome: illustrative cases and guidelines for prevention and treatment. Eur J Clin Nutr 2008;62:687-94

20. Peng M, Liu Y, Zhang XQ, et al. CTRP5-Overexpression Attenuated Ischemia-Reperfusion Associated Heart Injuries and Improved Infarction Induced Heart Failure. Front Pharmacol 2020;11:603322.

21. Wu X, Kong Q, Xia Z, et al. Penehyclidine hydrochloride alleviates lipopolysaccharide-induced acute lung injury in rats: Potential role of caveolin-1 expression upregulation. Int J Mol Med 2019;43:2064-74.

22. Zhao H, Chen H, Xiaoyin M, et al. Autophagy Activation Improves Lung Injury and Inflammation in Sepsis. Inflammation 2019;42:426-39.

23. Doig GS, Simpson F, Heighes PT, et al. Restricted versus continued standard caloric intake during the management of refeeding syndrome in critically ill adults: a randomised, parallel-group, multicentre, single-blind controlled trial. Lancet Respir Med 2015;3:943-52.

24. Olthof LE, Koekkoek WACK, van Setten C, et al. Impact of caloric intake in critically ill patients with, and without, refeeding syndrome: A retrospective study. Clin Nutr 2018;37:1609-17.

25. Tang QQ, Hong ZW, Ren HJ, et al. Nutritional Management of Patients With Enterocutaneous Fistulas: Practice and Progression. Front Nutr 2020;7:564379.

26. Singer P, Blaser AR, Berger MM, et al. ESPEN guideline on clinical nutrition in the intensive care unit. Clin Nutr 2019;38:48-79.

27. da Silva JSV, Seres DS, Sabino K, et al. ASPEN Consensus Recommendations for Refeeding Syndrome. Nutr Clin Pract 2020;35:178-95.

28. Melo DS, Costa-Pereira LV, Santos CS, et al. Severe Calorie Restriction Reduces Cardiometabolic Risk Factors and Protects Rat Hearts from Ischemia/Reperfusion Injury. Front Physiol 2016;7:106.

29. Salles J, Cardinault N, Patrac V, et al. Bee pollen improves muscle protein and energy metabolism in malnourished old rats through interfering with the Mtor signaling pathway and mitochondrial activity. Nutrients 2014;6:5500-16.

30. Kawamura H, Tanaka S, Uenami Y, et al. Hypophosphatemia occurs with insulin administration during refeeding by total parenteral nutrition in rats. J Med Invest 2018;65:50-5.
31. Reber E, Friedli N, Vasiloglou MF, et al. Management of Refeeding Syndrome in Medical Inpatients. J Clin Med 2019;8:2202.

32. Khan LU, Ahmed J, Khan S, et al. Refeeding syndrome: a literature review. Gastroenterol Res Pract 2011;2011:410971.

33. Boateng AA, Sriram K, Meguid MM, et al. Refeeding syndrome: treatment considerations based on collective analysis of literature case reports. Nutrition 2010;26:156-67.

34. Lin X, Lin CH, Liu R, et al. Myricetin against myocardial injury in rat heat stroke model. Biomed Pharmacother 2020;127:110194.

35. Zong L, Wang W. CircANXA2 Promotes Myocardial Apoptosis in Myocardial Ischemia-Reperfusion Injury via Inhibiting miRNA-133 Expression. Biomed Res Int 2020;2020:8590861.

36. Ulland TK, Song WM, Huang SC, et al. TREM2 Maintains Microglial Metabolic Fitness in Alzheimer's Disease. Cell 2017;170:649-663.e13.

37. Ferro F, Servais S, Besson P, et al. Autophagy and mitophagy in cancer metabolic remodelling. Semin Cell Dev Biol 2020;98:129-38.

38. Maejima Y, Isobe M, Sadoshima J. Regulation of autophagy by Beclin 1 in the heart. J Mol Cell Cardiol 2016;95:19-25.

39. Kaludercic N, Maiuri MC, Kaushik S, et al. Comprehensive autophagy evaluation in cardiac disease models. Cardiovasc Res 2020;116:483-504.

40. Chen X, Sun Y, Wang B, et al. Prognostic significance of autophagy-related genes Beclin1 and LC3 in ovarian cancer: a meta-analysis. J Int Med Res 2020;48:300060520968299.

41. Yang P, Li Z, Tye KD, et al. Effects of an orally supplemented probiotic on the autophagy protein LC3 and Beclin 1 in placentas undergoing spontaneous delivery during normal pregnancy. BMC Pregnancy Childbirth 2020;20:216.

42. Morel J, Hargreaves I, Brealey D, et al. Simvastatin pretreatment improves survival and mitochondrial function in a 3-day fluid-resuscitated rat model of sepsis. Clin Sci (Lond) 2017;131:747-58.

43. Yang M, Linn BS, Zhang Y, et al. Mitophagy and mitochondrial integrity in cardiac ischemiareperfusion injury. Biochim Biophys Acta Mol Basis Dis 2019;1865:2293-302.

44. Rocha AG, Franco A, Krezel AM, et al. MFN2 agonists reverse mitochondrial defects in preclinical models 


\section{Page 14 of 14}

of Charcot-Marie-Tooth disease type 2A. Science 2018;360:336-41.

45. Lazarou M, Sliter DA, Kane LA, et al. The ubiquitin kinase PINK1 recruits autophagy receptors to induce mitophagy. Nature 2015;524:309-14.

46. Nguyen TN, Padman BS, Lazarou M. Deciphering the Molecular Signals of PINK1/Parkin Mitophagy. Trends Cell Biol 2016;26:733-44.

47. Liu W, Chen C, Gu X, et al. AM1241 alleviates myocardial ischemia-reperfusion injury in rats by enhancing Pink1/ Parkin-mediated autophagy. Life Sci 2021;272:119228.

48. Kanwal A, Pillai VB, Samant S, et al. The nuclear and mitochondrial sirtuins, Sirt6 and Sirt3, regulate each other's activity and protect the heart from developing obesity-mediated diabetic cardiomyopathy. FASEB J 2019;33:10872-88.

49. Parodi-Rullán RM, Chapa-Dubocq XR, Javadov S. Acetylation of Mitochondrial Proteins in the Heart: The Role of SIRT3. Front Physiol 2018;9:1094.

50. Zheng Y, Shi B, Ma M, et al. The novel relationship between Sirt3 and autophagy in myocardial ischemia-

Cite this article as: Li J, Lu K, Zhang X, Wang T, Li Q, Yu X, Han W, Sun L. SIRT3-mediated mitochondrial autophagy in refeeding syndrome-related myocardial injury in sepsis rats. Ann Transl Med 2022;10(4):211. doi: 10.21037/atm-22-222
Li et al. Mitochondrial autophagy in RFS-related myocardial injury

reperfusion. J Cell Physiol 2019;234:5488-95.

51. Kane AE, Sinclair DA. Sirtuins and NAD+ in the Development and Treatment of Metabolic and Cardiovascular Diseases. Circ Res 2018;123:868-85.

52. Yu W, Gao B, Li N, et al. Sirt3 deficiency exacerbates diabetic cardiac dysfunction: Role of Foxo3A-Parkinmediated mitophagy. Biochim Biophys Acta Mol Basis Dis 2017;1863:1973-83.

53. Wu J, Yang Y, Gao Y, et al. Melatonin Attenuates Anoxia/ Reoxygenation Injury by Inhibiting Excessive Mitophagy Through the MT2/SIRT3/FoxO3a Signaling Pathway in H9c2 Cells. Drug Des Devel Ther 2020;14:2047-60.

54. M Parodi-Rullán R, Chapa-Dubocq X, GuzmánHernández R, et al. The Role of Adenine Nucleotide Translocase in the Assembly of Respiratory Supercomplexes in Cardiac Cells. Cells 2019;8:1247.

55. Hoshino A, Wang WJ, Wada S, et al. The ADP/ATP translocase drives mitophagy independent of nucleotide exchange. Nature 2019;575:375-9.

(English Language Editor: C. Mullens) 